\title{
Growth arrest and apoptosis induction in androgen receptor- positive human breast cancer cells by inhibition of USP14-mediated androgen receptor deubiquitination
}

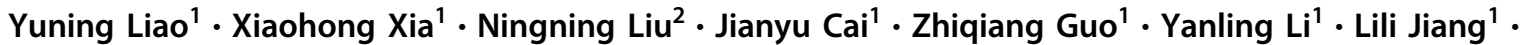 \\ Q. Ping Dou ${ }^{1,3,4} \cdot$ Daolin Tang $^{5,6} \cdot$ Hongbiao Huang $^{1} \cdot$ Jinbao Liu ${ }^{1}$
}

Received: 2 June 2017 / Revised: 23 August 2017 / Accepted: 19 September 2017 / Published online: 22 January 2018

(c) The Author(s) 2018. This article is published with open access

\begin{abstract}
It has been well known that androgen receptor (AR) is critical to prostate cancer development and progression. It has also been documented that AR is expressed in more than $60 \%$ of breast tumors, which promotes the growth of estrogen receptornegative $\left(\mathrm{ER}^{-}\right) / \mathrm{AR}$-positive $\left(\mathrm{AR}^{+}\right)$breast cancer cells. Thus, $\mathrm{AR}$ might be a potential therapeutic target for AR-positive/ERnegative breast cancer patients. Previously we reported that in prostate cancer cells proteasome-associated deubiquitinase ubiquitin-specific protease 14 (USP14) stabilized AR protein level by removing its ubiquitin chain. In the current study, we studied the USP14-AR protein interaction and cell proliferation status after USP14 reduction or inhibition in breast cancer cells, and our results support the conclusion that targeting USP14 is a novel strategy for treating AR-responsive breast cancer. We found that inhibition of USP14 accelerated the K48-ubiquitination and proteasome-mediated degradation of AR protein. Additionally, both genetic and pharmacological inhibition of USP14 significantly suppressed cell proliferation in AR-responsive breast cancer cells by blocking $G_{0} / G_{1}$ to $S$ phase transition and inducing apoptosis. Moreover, AR overexpression inhibited USP14 inhibition-induced events, suggesting that AR deubiquitination by USP14 is critical for breast cancer growth and USP14 inhibition is a possible strategy to treat AR-positive breast cancer.
\end{abstract}

\section{Introduction}

Breast cancer, an increasing threat to women in the world, is considered as a heterogeneous disease. Breast cancer can be divided into three major subtypes, based on the status of

Yuning Liao, Xiaohong Xia and Ningning Liu contributed equally to this work.

Hongbiao Huang

hhb800616@126.com

$\triangle$ Jinbao Liu

jliu@gzhmu.edu.cn

1 Affiliated Cancer Hospital of Guangzhou Medical University; Protein Modification and Degradation Lab, SKLRD, School of Basic Medical Sciences, Guangzhou Medical University, Guangzhou, Guangdong, China

2 Guangzhou Institute of Cardiovascular Disease, the Second Affiliated Hospital, Guangzhou Medical University, Guangzhou, Guangdong, China estrogen receptor (ER), progestogen receptor (PR), and HER2 status [1, 2]. Sex steroid hormones are critical to the growth and development of the uterus and breast/prostate in women/men [3]. Estrogen/ER is a highly attractive target for anti-breast cancer strategies; however, current endocrine therapies are ineffective for $25-30 \%$ of ER-negative $\left(\mathrm{ER}^{-}\right)$ breast cancers. Therefore, identifying new, novel therapeutic targets in advanced $\mathrm{ER}^{-}$breast cancer is critical and urgent.

3 The Molecular Therapeutics Program, Barbara Ann Karmanos Cancer Institute, Detroit, MI, USA

4 Departments of Oncology, Pharmacology and Pathology, School of Medicine, Wayne State University, Detroit, MI, USA

5 The Third Affiliated Hospital, Center for DAMP Biology, Key Laboratory for Major Obstetric Diseases of Guangdong Province, Key Laboratory of Reproduction and Genetics of Guangdong Higher Education Institutes, Protein Modification and Degradation Laboratory, Guangzhou Medical University, Guangzhou, Guangdong, China

6 Department of Surgery, University of Pittsburgh, Pittsburgh, PA, USA 
Fig. 1 USP14 inhibition or silencing downregulates AR protein level in breast cancer cells. a Protein lysates were collected from the indicated breast cancer cells. Western blot assay was used to detect the expression of USP14 and AR proteins. GAPDH was used as an internal control. b Protein lysates were collected from the indicated breast cancer cells stably expressing USP14 shRNA or control shRNA. Western blot assay was used to detect the expression of USP14, GFP, and AR proteins. c Protein lysates were collected from the indicated breast cancer cells treated with the indicated concentrations of IU1 for $48 \mathrm{~h}$. Western blot assay was used to detect the expression of USP14 and AR proteins. d MDA-MB453 cells stably expressing USP14 or control shRNA or treated with IU1 or DMSO, and then treated with cycloheximide (CHX) for various lengths of time. Western blot assay was used to detect AR and USP14 protein level

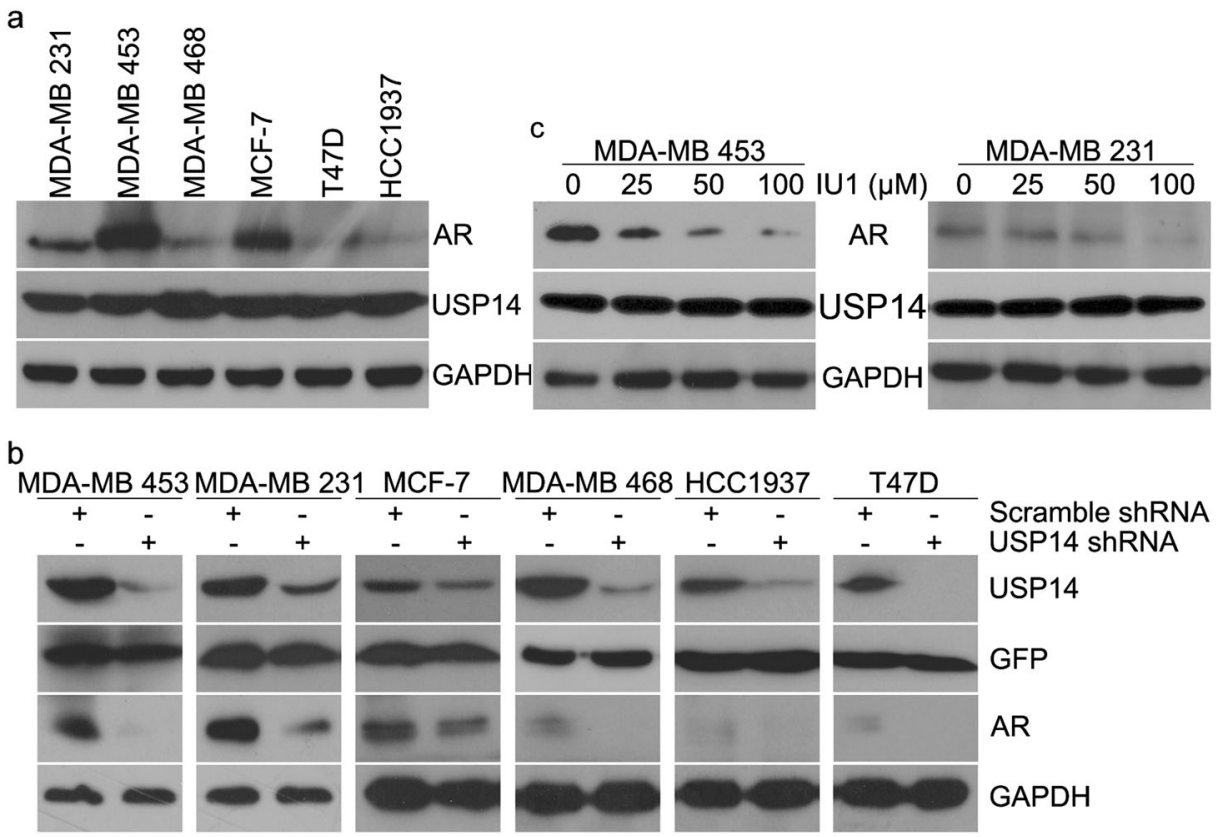

d

$\mathrm{CHX} 50 \mu \mathrm{g} / \mathrm{ml}$

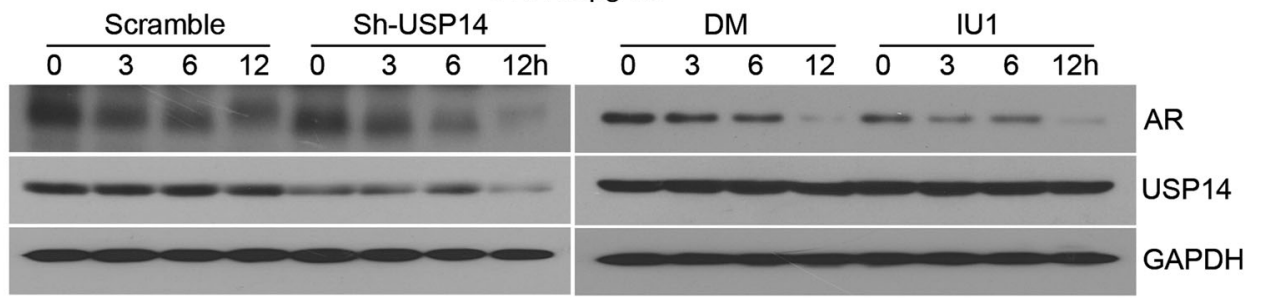

Expression of $\mathrm{AR}$ is a phenomenon in most breast tumors, irrespective of ER status [4,5]. Although AR has an anti-proliferative effect in $\mathrm{ER}^{+}$breast cancer by antagonizing ER [6], recent studies have also shown that AR facilitates the proliferation of $\mathrm{ER}^{-}$breast carcinomas $[3,7$, 8]. Indeed, AR signaling inhibitors, small molecules that bind $\mathrm{AR}$ and inhibit its nuclear translocation, such as enzalutamide and bicalutamide, have shown some efficacy in treating advanced $\mathrm{ER}^{-} / \mathrm{AR}^{+}$breast cancer, which established a potential strategy for treating anti-ER ${ }^{-} / \mathrm{AR}^{+}$breast cancer by targeting AR [7, 9]. Recent study also shows that the level of AR expression may represent a valuable prognostic marker or tool for treatment selection in breast cancer [10]. These findings collectively suggest that androgens may promote breast carcinogenesis, and AR could be developed as a therapeutic target for breast cancers.

$\mathrm{AR}$ is highly regulated by the ubiquitin proteasome system. Ubiquitination of AR may affect or even change its function and location, or promote its degradation; AR ubiquitination can be reversed by deubiquitination mediated by deubiquitinases (DUBs) [11]. Increasing levels of E3 ubiquitin ligases, such as MDM2, CHIP, and SIAH2, have been shown to co-regulate $\mathrm{AR}$ and therefore control AR stability and activity [12-14]. The function of DUBs, key effectors of deubiquitination, is to remove mono-ubiquitin (Ub) or poly-Ub chains from target proteins, resulting in protein degradation or prevention of degradation, and by doing so, DUBS are involved in the regulation of multiple cellular processes. Indeed, several DUBs, including USP26, USP12, USP10, and USP7, have been reported to interact with AR protein and overcome the Ub-ligase effects of MDM2, CHIP, or SIAH2 [15-19].

There are three DUBs, USP14, UCHL5, and Rpn11 (POH1) present in mammalian 19 S proteasome complexes. Rpn11 is an intrinsic subunit of $19 \mathrm{~S}$ regulatory particle, whereas USP14 and UCHL5 reversibly associate with $19 \mathrm{~S}$ proteasome, indicative of attractive and versatile roles for these DUBs [20-22]. As a member of the ubiquitin-specific processing protease (USP) family, USP14 has been reported to be overexpressed in various cancers, including multiple myeloma, ovarian carcinoma, and colorectal cancer [2224]. Different from numerous DUBs, the deubiquitinating activity of USP14 is activated by proteasome [25-27]. USP14 counteracts the function of proteasome by mediating 

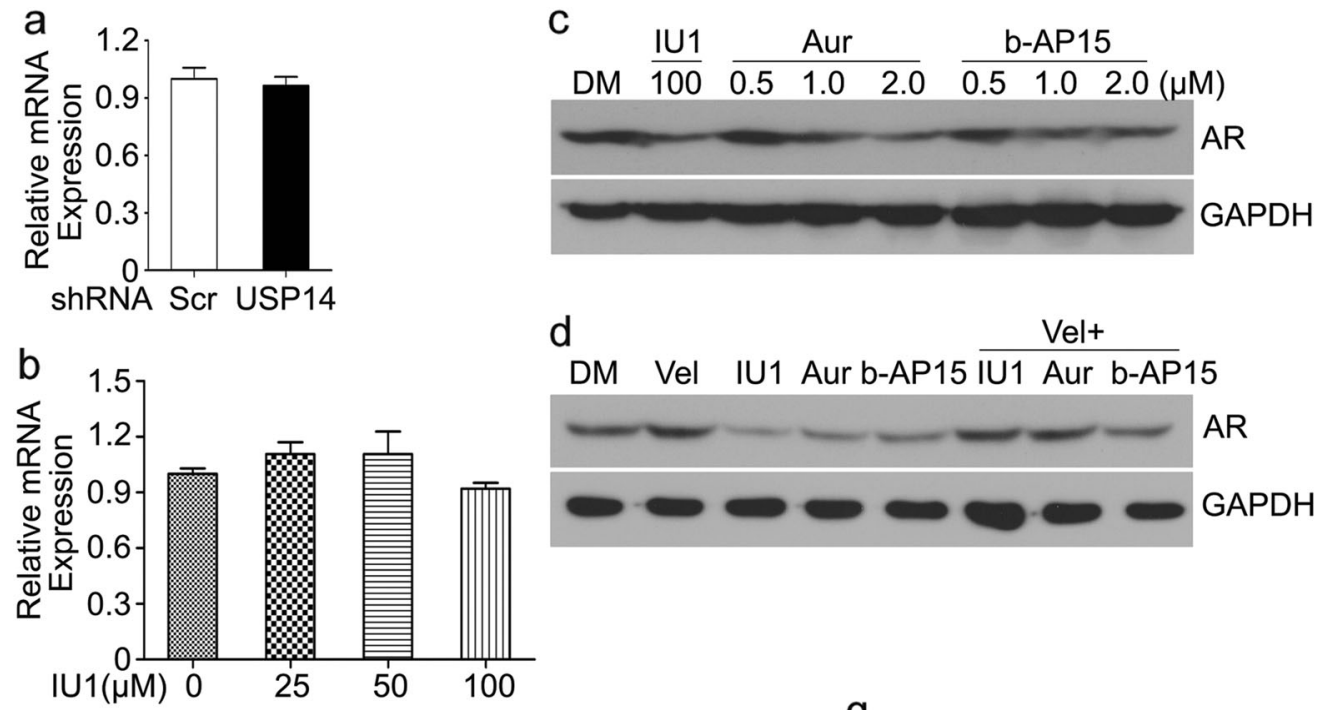

d

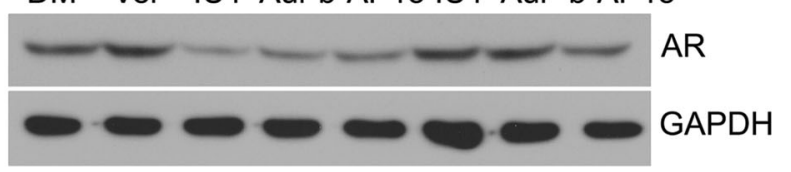

e IP $\quad f$ Input $\overline{\lg G \text { AR }}$ $\begin{aligned} & \text { IB: AR } \\ & \text { IB: USP14 } \\ & \text { IB: UCHL5 }\end{aligned}$

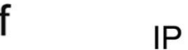

$g_{\text {LNcap MDA-MB } 453}$

Input IgG USP14 $++\quad+\quad+\quad-$ Scramble siRNA
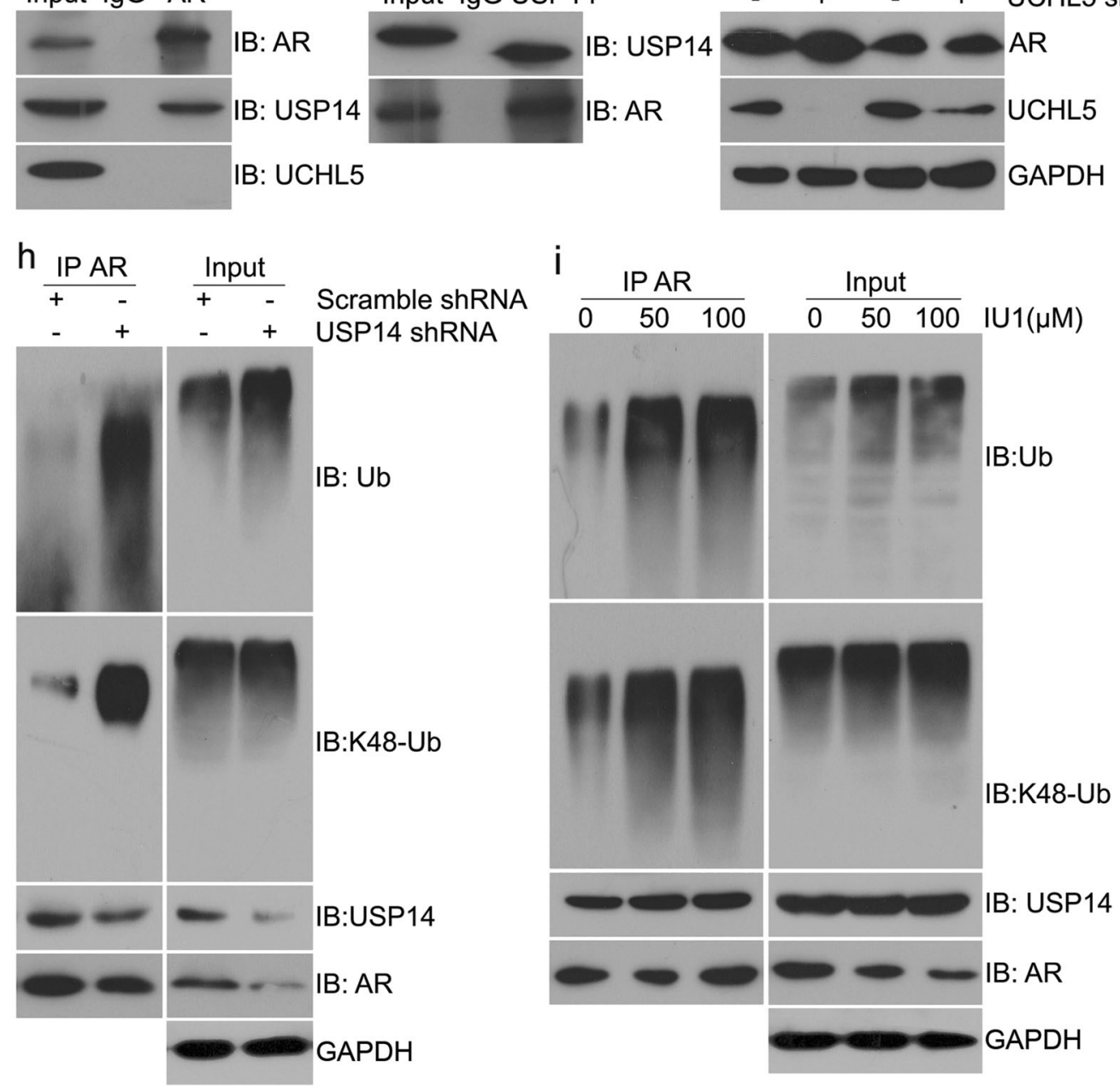
Fig. 2 Loss of USP14 expression/function accumulates the poly-K48ubiquitination of AR and promotes AR degradation. a Total RNAs were collected from MDA-MB-453 cells stably expressing USP14 shRNA or control shRNA and subjected to RT ${ }^{2}$-PCR analysis. Three independent experiments were performed. Mean \pm S.D. $(n=3)$. b Total RNAs were extracted from MDA-MB-453 cells treated with IU1 for $24 \mathrm{~h}$ and subjected to $\mathrm{RT}^{2}$-PCR analysis. Three independent experiments were performed. Mean \pm S.D. $(n=3)$. c Protein lysates were collected from MDA-MB-453 cells treated with the indicated doses of IU1, auranofin (Aur), or b-AP15 for $48 \mathrm{~h}$. Western blot assay was used to detect AR protein level. d Protein lysates were collected from MDA-MB-453 cells exposed to IU1 $(100 \mu \mathrm{M})$, Aur $(1 \mu \mathrm{M})$, and b-AP15 $(1 \mu \mathrm{M})$ in the presence or absence of bortezomib/ Velcade (50 $\mathrm{nM})$ for $24 \mathrm{~h}$ and western blot assay was used to detect $\mathrm{AR}$ protein level. e, f Protein lysates were collected from MDA-MB-453 cells. Coimmunoprecipitation assay was performed to detect AR, USP14, and UCHL5 interaction. g Protein lysates were collected from LNCaP and MDA-MB-453 cells treated with UCHL5 siRNA for $48 \mathrm{~h}$. Western blot assay was used to detect AR and UCHL5 protein level. h Protein lysates were collected from MDA-MB-453 cells stably expressing USP14 shRNA or control shRNA. Co-immunoprecipitation assay was performed using AR antibody beads, and immunoblotted for ubiquitin (Ub), K48-Ub, USP14, and AR. Cells were exposed to MG132 (10 $\mu \mathrm{M})$ for $6 \mathrm{~h}$ before harvest. i Protein lysates were collected from MDA-MB-453 cells treated with IU1 for $48 \mathrm{~h}$. Coimmunoprecipitation assay was performed using AR antibody beads, and immunoblotted for ubiquitin (Ub), K48-Ub, USP14, and AR. Cells were exposed to MG132 $(10 \mu \mathrm{M})$ for $6 \mathrm{~h}$ before harvest

rapid deubiquitination and reducing the anchoring time of ubiquitin conjugates, and thereby suppressing the degradation of the substrate proteins [27, 28]. Previously we found that the proteasome-associated DUB USP14 promoted the cell cycle in prostate carcinoma cells by deubiquitination and stabilization of AR [11]. The current study demonstrated that USP14 is crucial for the growth and survival of $\mathrm{AR}^{+} / \mathrm{ER}^{-}$breast cancer, which was dependent on $\mathrm{AR}$ status.

\section{Results}

\section{USP14 regulates AR protein level in breast cancer}

In the current study, we first determined the expression levels of AR and USP14 proteins in six breast cancer cell lines using Western blot analysis. We observed high expression of USP14 protein in all six breast cancer cell lines (Fig. 1a). However, AR expression was higher in MDA-MB-453, MDA-MB-231, and MCF7 cell lines than in MDA-MB-468, HCC1937, and T47D lines (Fig. 1a). Therefore, profile of USP14 expression is not positively correlated with that of AR. This phenomenon is similar to our previous findings from prostate cancer cell lines [11], which might suggest a potential role of USP14 in the progression of breast carcinomas. We previously reported that USP14 regulates the expression or function of AR in prostate cancer cells [11]. We then applied USP14 short hairpin RNA (shRNA) in breast cancer cells to knockdown
USP14 and studied the consequent effect on the AR expression; we found that inhibition of USP14 expression by its specific shRNA treatment caused great decrease in AR expression in $\mathrm{AR}^{+}$breast cancer cells (Fig. 1b). To verify whether this shRNA effect is dependent on inhibition of the deubiquitinating activity of USP14, we used IU1, a potent pharmacological inhibitor that selectively inhibits USP14 by preventing its docking on the proteasome [27], and tested the effect of USP14 inhibition by IU1 on the AR expression in breast cancer cells. We found that similar to USP14 shRNA (Fig. 1b), inhibition of USP14 by IU1 also significantly decreased AR protein level in breast cancer cells (Fig. 1c), supporting the conclusion that proteasomal DUB USP14 regulates the expression of AR in breast cancer cells. To determine whether USP14 regulates the stability of AR protein, we used cycloheximide (CHX). Treatment of control MDA-MB-453 (either scramble shRNA or parental) cells with CHX for up to $12 \mathrm{~h}$ caused decreased levels of AR (Fig. 1d), suggesting a contribution of AR protein synthesis to endogenous AR protein levels; however, co-treatment of CHX and USP14 shRNA or IU1 resulted more rapid decrease in levels of endogenous $A R$ protein (Fig. 1d), strongly suggest that deubiquitination of AR protein by USP14 is essential for its protein stability.

\section{USP14 interacts with, and stabilizes AR protein}

It has been shown that DUBs regulate their substrate proteins on multiple levels, including post-translational and protein-protein interaction. DUBs can directly bind to target proteins, and can also interact with transcription factors or histone-associated proteins, thereby regulating transcription [29]. Thus, we investigated whether USP14 regulates the transcription of AR. Data from RT-PCR assay demonstrated that the mRNA level of AR was not affected by either genetic or pharmacological inhibition of USP14 (Fig. 2a, b). We therefore hypothesized that inhibition or silencing of USP14 could induce AR downregulation through enhancing AR degradation. To test effects of other inhibitors that potently inhibit both USP14 and UCHL5, we used auranofin (Aur) [30] or b-AP15 [31] and determined if they could affect AR expression in breast cancer MDA-MB453 cells. We found that both Aur and b-AP15 decreased the expression of AR in a dose-dependent fashion (Fig. 2c), and the reduction can be rescued by using bortezomib (Velcade/Vel), a selective $20 \mathrm{~S}$ proteasome inhibitor (Fig. 2d).

To determine if there is any interaction between AR protein and USP14 or UCHL5 protein, we performed coimmunoprecipitation (co-IP) for AR, USP14, and UCHL5. We found that USP14, but not UCHL5, directly binds AR protein (Fig. 2e, f). In addition, reduction of UCHL5 by its siRNA did not affect the expression of AR (Fig. 2g), 

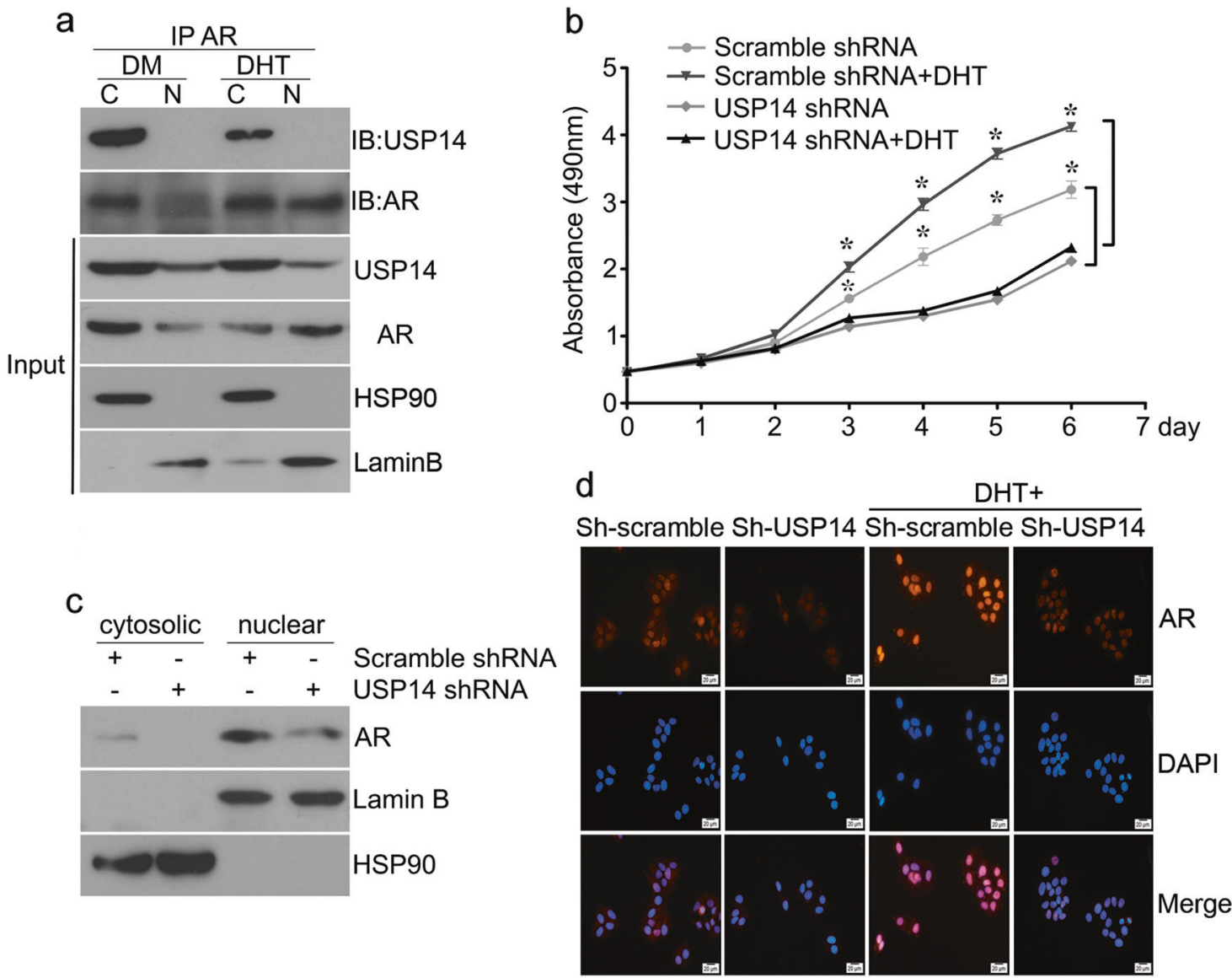

Fig. 3 USP14 in the cytoplasm is critical to the nuclear import of AR. a MDA-MB-453 cells were treated with DHT $(10 \mathrm{nM})$ for $24 \mathrm{~h}$. Cytosolic and nuclear protein fractions were prepared, which were then immunoprecipitated with AR antibody beads, and immunoblotted for AR and USP14. HSP90 was used as a cytoplasmic control. Lamin B was used as a nuclear control. b MDA-MB-453 cells stably expressing USP14 shRNA or control shRNA were exposed to DHT $(10 \mathrm{nM})$ for indicated days. Cell viability was detected using MTS assay. Error bars correspond to a $95 \% \mathrm{CI}$ of three independent

suggesting that USP14 but not UCHL5 recruited on the19S proteasome plays a selective role in the deubiquitination of AR. To further confirm that USP14 is a DUB of AR, we determined the effect of IU1 or USP14 shRNA on the abundance of poly-ubiquitinated and K48-polyubiquitinated AR using co-IP. We found that IU1 and USP14 knockdown dramatically increased levels of ubiquitinated and K48-ubiquitinated AR (Fig. 2h, i), suggesting that USP14 is an AR DUB, capable of deubiquitinating and thereby stabilizing AR protein.

\section{USP14 is not required for AR translocation}

DUBs could regulate the localization or function of target proteins. USP10 is a bona fide DUB that deubiquitinates experiments. ${ }^{*} P<0.05$. c Cytosolic and nuclear protein lysates were extracted, respectively, from MDA-MB-453 cells stably expressing USP14 or control shRNA exposed to DHT $(10 \mathrm{nM})$ for $24 \mathrm{~h}$. Western blot assay was used to detect AR expression. d MDA-MB-453 cells stably expressing USP14 shRNA or control shRNA were exposed to vehicle (Veh) or DHT $(10 \mathrm{nM})$ for $24 \mathrm{~h}$. Immunofluorescence microscopy shows endogenous AR (orange) and nucleus (blue). Scale bars represent $20 \mu \mathrm{m}$

p53 and AR in the cytoplasm and enhances their nuclear import and transcriptional activity [19, 32]. Whether USP14 could co-translocate with AR into the nucleus is unclear. To determine the interaction between AR and USP14 protein in the nucleus, we performed co-IP for AR and USP14 using both nuclear and cytosolic preparations of MDA-MB-453 cells treated with DHT or control solvent for $24 \mathrm{~h}$. We found that USP14 does not interact with AR in the nucleus under DHT stimulation (Fig. 3a). In addition, western blot analysis and immunofluorescent staining assay indicated that USP14 silencing significantly downregulated the abundance of AR in both the nucleus and cytoplasm under androgen stimulation (Fig. 3c, d), suggesting that cytosolic USP14 is not required for AR translocation and that USP14 silencing-induced decrease of nuclear AR could be due to 

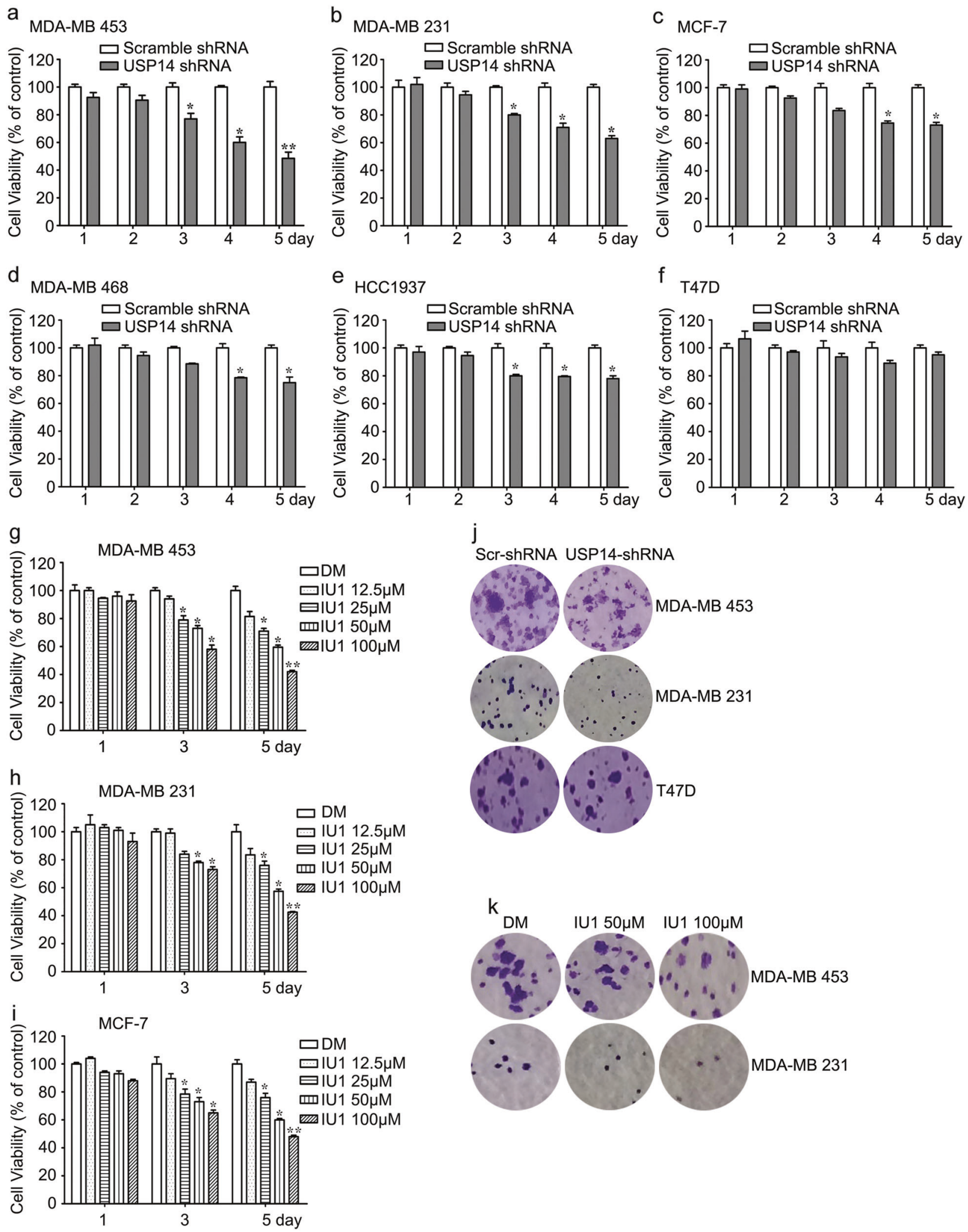

Fig. 4 USP14 inhibition or silencing suppresses the proliferation of $\mathrm{AR}^{+}$breast tumor cells. a-f MDA-MB-453, MDA-MB-231, MCF7, MDA-MB-468, HCC1937, and T47D cells were exposed to USP14 shRNA daily for up to 5 days. g-i MDA-MB-453, MDA-MB231, and MCF7 cells were exposed to IU1 for 1, 3, and 5 days. Cell viability was detected using MTS assay. Error bars correspond to $95 \%$

CI of three independent experiments. ${ }^{*} P<0.05, * * P<0.01$ vs. each vehicle control. $\mathbf{j}$ Colony formation assay was performed in the indicated breast cancer cells stably expressing control shRNA or USP14 shRNA for 2 weeks; representative images are shown. $\mathbf{k}$ Colony formation assay was performed in the indicated breast cancer cells exposed to IU1 for 2 weeks; representative images are shown 

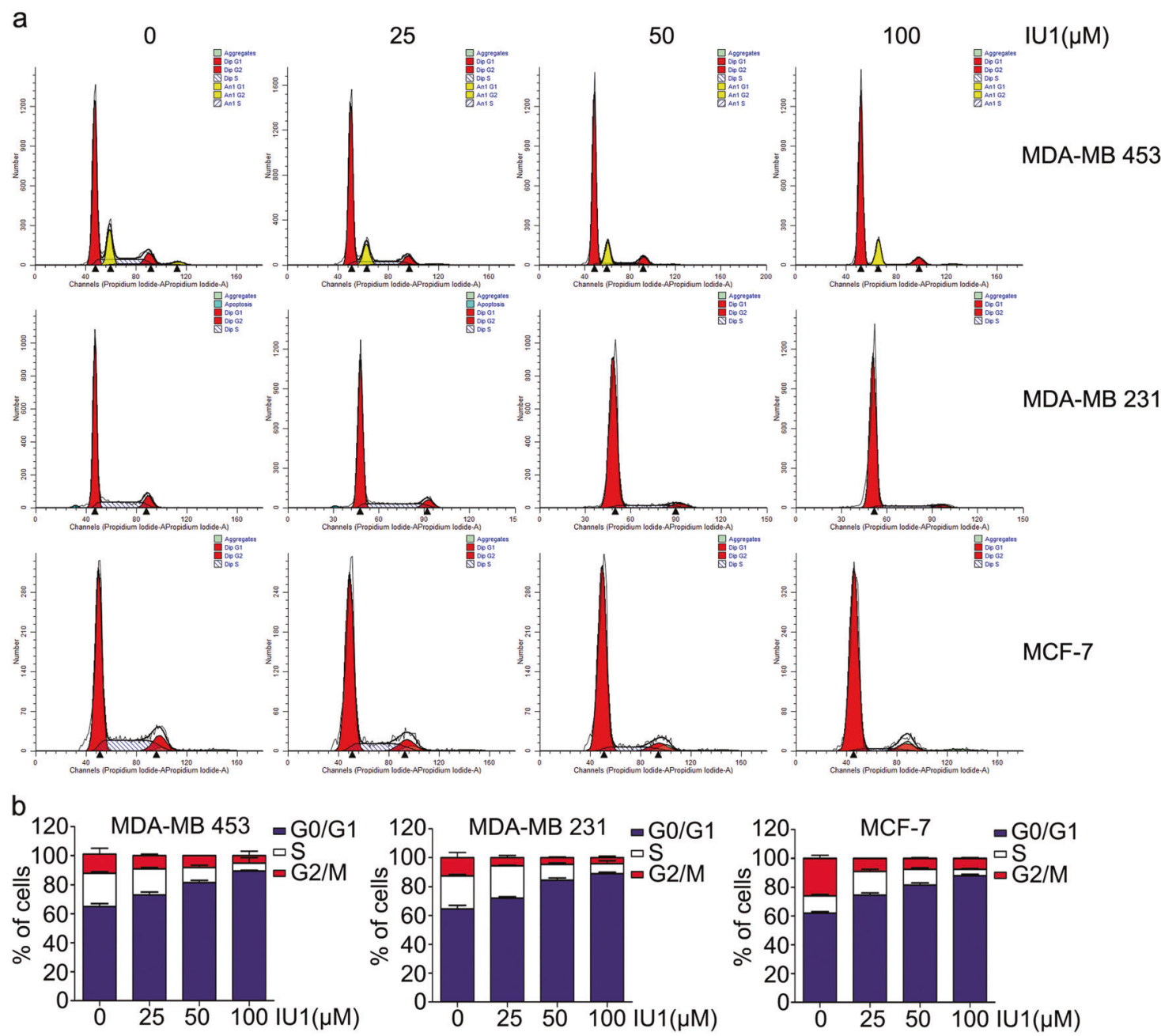

b
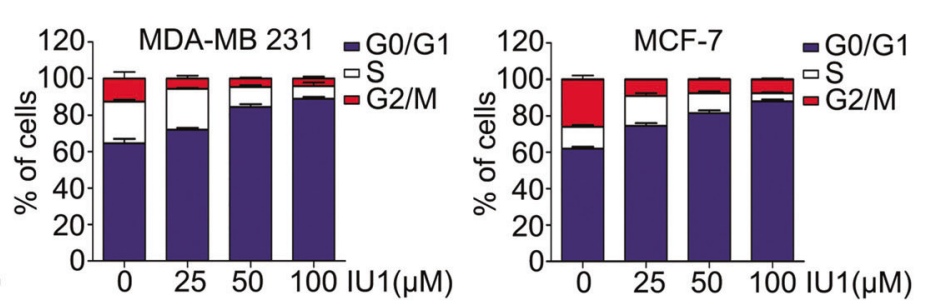

C
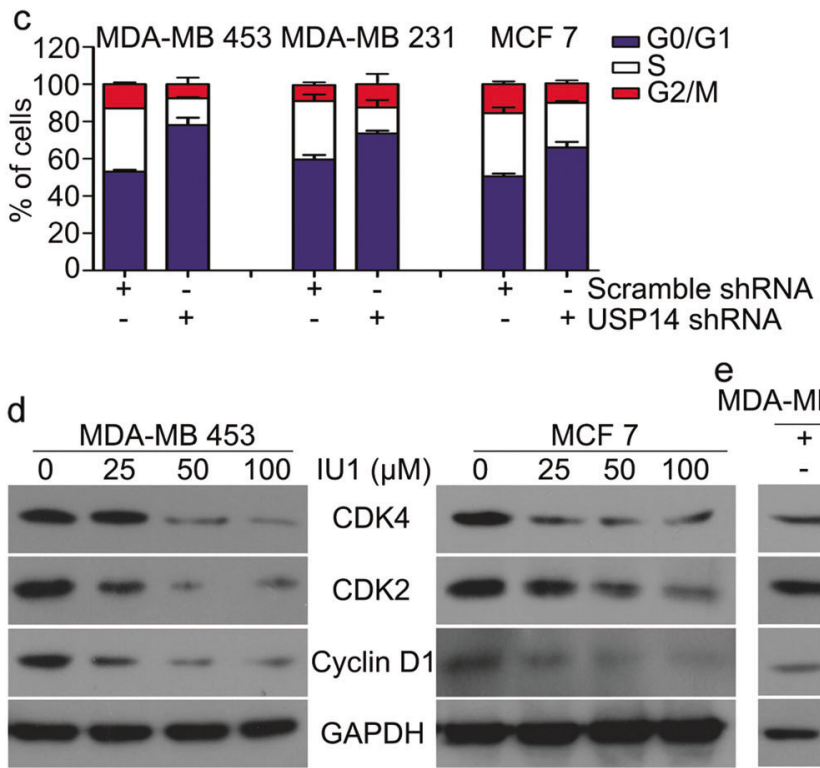

e

MDA-MB 453 MDA-MB 231 MCF-7

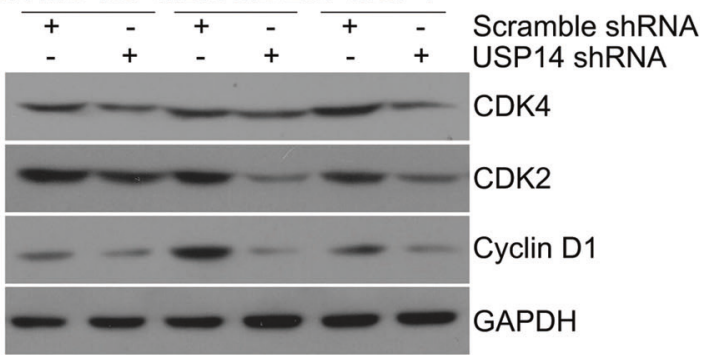


Fig. 5 USP14 inhibition or silencing blocks $G_{0} / G_{1}$ to $S$ phase transition in breast cancer cells. a Fluorescence-activated cell sorting analysis (FACS) was performed on the indicated breast cancer cells exposed to IU1 for $48 \mathrm{~h}$. $(n=3)$. b The percentage of cells in each population was calculated. Mean \pm S.D. $(n=3)$. c FACS analysis was performed on the indicated breast cancer cells that stably expressed USP14 shRNA or control shRNA for $48 \mathrm{~h}$. The percentage of cells in each population was calculated. Mean \pm S.D. $(n=3)$. d Protein lysates were collected from the indicated breast cancer cells treated with IU1 for $48 \mathrm{~h}$. Western blot assay was used to detect CDK4, CDK2, and cyclin D1 protein expression. GAPDH was used as an internal control. e Protein lysates were collected from the indicated breast cancer cells stably expressing USP14 shRNA or control shRNA. Western blot assay was used to detect CDK4, CDK2, and cyclin D1 expression. GAPDH was used as an internal control

the decrease of total AR protein. To investigate the effect of USP14 knockdown on DHT-mediated events in MDA-MB453 cells, we compared the proliferation rates of USP14 shRNA knockdown or control cells after treatment with DHT for up to 7 days using MTS assay. We found that DHT promoted the proliferation of the control group, but had little enhancing effect on the proliferation of the USP14 knockdown group (Fig. 3b). Collectively, the results show that USP14 regulates the total AR level but not AR translocation, and mediates the responsiveness of $\mathrm{AR}^{+} / \mathrm{ER}$ breast cancer to androgen.

\section{Inhibition of USP14 suppresses the proliferation of breast cancer}

To study the potential role of USP14 in the proliferation of breast tumor cells, we determined the effect of USP14 knockdown on breast cell viability. We found that USP14 inhibition by shRNA significantly suppressed the proliferation of MDA-MB-231, MDA-MB-453, MDA-MB468, HCC1937, and MCF7 breast cancer cell lines (Fig. 4ae); however USP14 knockdown did not affect the proliferation of T47D cells (Fig. 4f), which could be related to the fact that these cells express very high levels of ER and very low levels of AR (Fig. 1a). In addition, we also used the pharmacological USP14 inhibitor IU1 $(12.5,25,50$, $100 \mu \mathrm{M})$ and tested the effect on the proliferation of breast cancer cells. Inhibition of USP14 by IU1 caused a concentration-dependent, significant suppression on the proliferation of MDA-MB-453, MDA-MB-231, and MCF7 carcinoma cells (Fig. 4g-i). To test the long-term effect of USP14 inhibition or silencing on breast carcinoma cells, we performed breast cancer cell colony formation assay using either IU1 treatment or USP14 shRNA-generated stable clones. USP14 inhibition by either IU1 or specific shRNA dramatically decreased the colony formation of MDA-MB453 and MDA-MB-231 cells after 2 weeks; however, the identical experimental conditions had little or no inhibitory effect on T47D colonies. (Fig. 4j, k).

\section{Inhibition of USP14 causes $G_{0} / G_{1}$ arrest and apoptosis in breast cancer cells}

We then studied the underlying mechanism by which USP14 regulates the growth of $\mathrm{AR}^{+}$breast cancer cells. We monitored the cell cycle progression of each group of breast cancer cells exposed to various concentrations of IU1 (25, $50,100 \mu \mathrm{M})$, and found that inhibition of USP14 activity dramatically induced $G_{0} / G_{1}$ cell cycle arrest after $48 \mathrm{~h}$, associated with decreased population in $\mathrm{S} / \mathrm{G}_{2} / \mathrm{M}$ phases (Fig. 5a, b). In addition, silencing USP14 expression with stable expression of shRNA also caused an arrest of cells at $\mathrm{G}_{0} / \mathrm{G}_{1}$ (Fig. 5c), indicating that USP14 regulates the $\mathrm{G}_{1}$ to $\mathrm{S}$ transition in androgen-responsive breast cancer cells. To explore the molecular mechanism by which USP14 regulates the $\mathrm{G}_{1}-\mathrm{S}$ transition, Western blot analysis was performed to detect levels of several $G_{1}$ and $S$ key players. We found that the IU1 treatment cause a decrease in the protein level of cyclin D1, CDK4, and CDK2, all of which have been shown to be essential for $\mathrm{G}_{0} / \mathrm{G}_{1}$ to $\mathrm{S}$ phase transition (Fig. 5d). In USP14-stable knockdown MDA-MB-231, MDA-MB-453, and MCF7 cells protein levels of cyclin D1, CDK4, and CDK2 were also decreased (Fig. 5e). Collectively, these results indicate that USP14 regulates levels of key cell cycle regulators cyclin D1, CDK4, and CDK2 and consequently the $\mathrm{G}_{1}-\mathrm{S}$ phase transition in breast cancer cells.

We then determined whether apoptosis induction is also involved in the growth inhibition by USP14 inhibition or knockdown in breast cancer cells. To do so, we performed several specific assays, including annexinV-fluorescein isothiocyanate (FITC)/propidium iodide (PI)-staining, PARP cleavage, and Bcl-2 protein expression using western blot analyses. Bcl-2 is an anti-apoptotic protein, while p89 cleavage fragment of PARP is a molecular marker of apoptosis. We found that USP14 inhibition significantly induced apoptosis (Fig. 6a-d), as evident by PARP cleavage, and downregulation of the anti-apoptotic protein Bcl2, in $\mathrm{AR}^{+} / \mathrm{ER}^{-}$breast cancer (Fig. 6e, f), and moderately induced apoptosis in $\mathrm{AR}^{+} / \mathrm{ER}^{+} \mathrm{MCF} 7$ cells (Fig. 6a-f).

\section{AR overexpression suppresses USP14 inhibition- induced events}

To study the functionality of $\mathrm{AR}$ deubiquitination by USP14, we first compared the effects of AR depletion in the $\mathrm{AR}^{+} / \mathrm{ER}^{-}$MDA-MB-453 cells to T47D cells that express high ER and low AR. We found that AR depletion by siRNA led to $\mathrm{G}_{1}$ arrest and apoptosis in MDA-MB-453, but not in T47D (Fig. 7a, b); consistently, AR depletion decreased levels of CDK4, CDK2, cyclin D1, and Bcl-2 in MDA-MB-453, but not T47D cells (Fig. 7c, d). Next, we determined if re-introduction of $\mathrm{AR}$ would rescue the 

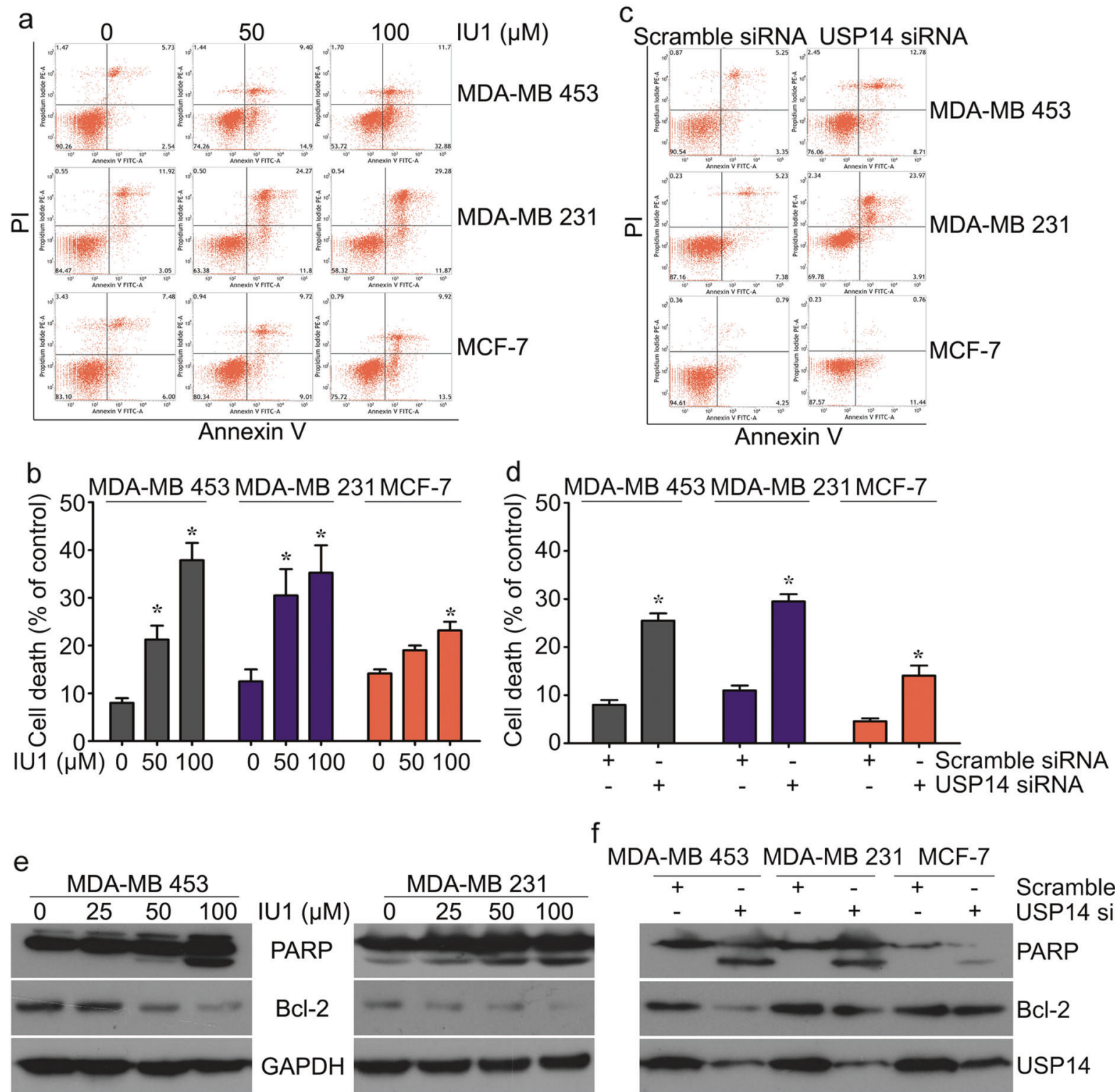

$\mathrm{f}$ MDA-MB 453 MDA-MB 231 MCF-7

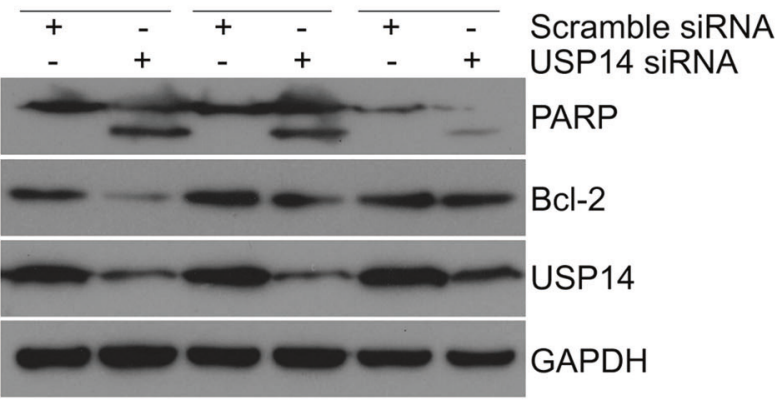

Fig. 6 USP14 inhibition or silencing induces apoptosis in $\mathrm{AR}^{+} / \mathrm{ER}^{-}$ breast cancer cells. The indicated breast cancer cell lines treated with IU1 for $72 \mathrm{~h}$ were collected. Flow cytometry analysis with annexinVFITC/PI staining was used to calculate the apoptotic cells. Representative images (a) and cell death population (b) from three independent replicates are shown. Mean \pm S.D. $(n=3)$. $* P<0.05$ vs. each vehicle control. The indicated breast cancer cells treated with USP14 siRNA or control siRNA for $72 \mathrm{~h}$ were collected. Flow cytometry analysis with annexinV-FITC/PI staining was used to calculate

growth inhibition induced by USP14 knockdown. To do so, MDA-MB-453 cells stably expressing control shRNA or USP14 shRNA were transfected with a tagged AR vector (HA-AR) or a control vector (Mock), followed by measurement of cell cycle progression. Overexpression of AR

the apoptotic cells. Representative images (c) and cell death population (d) are shown. Mean \pm S.D. $(n=3)$. $* P<0.05$ vs. each vehicle control. e Protein lysates were collected from the indicated breast cancer cells treated with IU1 for $48 \mathrm{~h}$. Western blot assay was used to detect PARP and Bcl-2 protein expression. f Protein lysates were collected from the indicated breast cancer cells treated with USP14 siRNA or control siRNA for $48 \mathrm{~h}$. Western blot assay was used to detect PARP and Bcl-2 expression. GAPDH was used as an internal control

was able to rescue cells from the USP14 silencing-induced $\mathrm{G}_{1}$ arrest (Fig. 7e). Western blot analysis from the same experiment confirmed increased levels of CDK4, CDK2, and Cyclin D1 protein after overexpressing AR (Fig. 7f). Additionally, AR overexpression inhibited cell apoptosis 

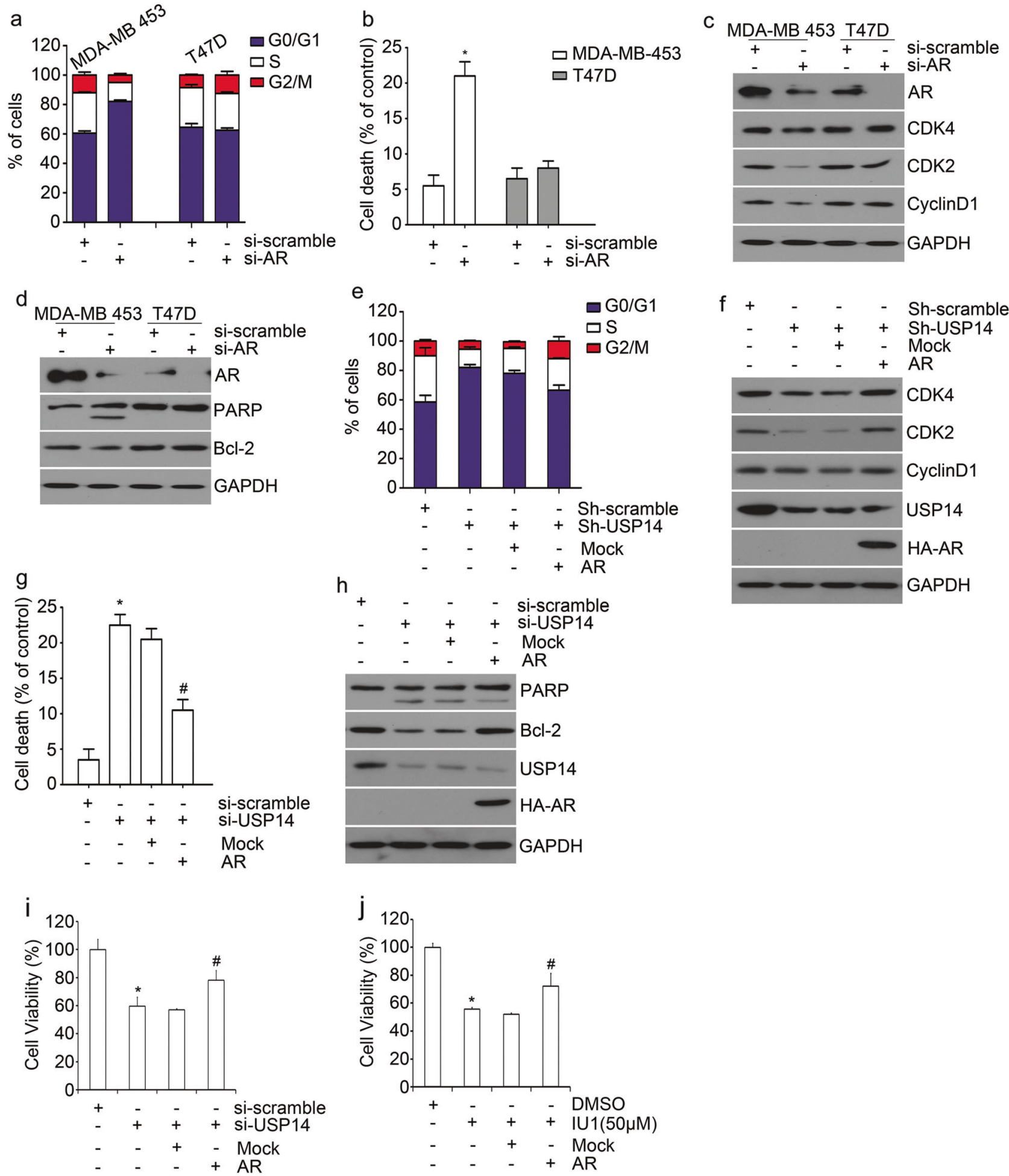

and growth suppression induced by USP14 silencing or IU1 treatment (Fig. 7g-j). Collectively, these findings demonstrate the functionality of AR deubiquitination by USP14 in promoting breast cancer cell proliferation.

\section{Discussion}

Breast cancer is a common threat to women's health in the whole world. ER, PR, and HER2 play important roles in promoting the development and progression of most breast 
Fig. 7 AR overexpression inhibits USP14 silencing-induced $G_{1}-S$ arrest and apoptosis. a Fluorescence-activated cell sorting analysis (FACS) was performed on the indicated breast cancer cells treated with the AR siRNA or control siRNA for $48 \mathrm{~h}$. Cells in each population were calculated from three independent replicates. Mean \pm S.D. $(n=3)$. b The indicated breast cancer cells treated with AR siRNA or control siRNA for $72 \mathrm{~h}$. Flow cytometry analysis with annexinVFITC/PI staining was used to calculate the apoptotic cells. Apoptotic populations from three independent replicates are shown. Mean \pm S.D. $(n=3) . * P<0.05$ vs. each vehicle control. $\mathbf{c}$ and $\mathbf{d}$ Protein lysates were collected from the indicated breast cancer cells exposed to AR siRNA or control siRNA for $48 \mathrm{~h}$. Western blot assay was used to detect the expression of AR, CDK4, CDK2, cyclin D1, PARP, and Bcl-2. e MDA-MB-453 cells stably expressing USP14 or control shRNA were transduced with AR or control vector for $48 \mathrm{~h}$ and subjected to FACS analysis. Cells in each population were calculated from three independent replicates. Mean \pm S.D. $(n=3)$. f Protein lysates were collected from MDA-MB-453 cells stably expressing USP14 or control shRNA transduced with AR or control vector for $48 \mathrm{~h}$. Western blot assay was used to detect the expression of CDK4, CDK2, cyclin D1, USP14, and HA-tag. g MDA-MB-453 cells exposed to USP14 siRNA or control siRNA with or without AR or control vector for $72 \mathrm{~h}$ were collected. Flow cytometry analysis with annexinV-FITC/ PI staining was used to calculate the apoptotic cells. Apoptotic populations from three independent replicates are shown. Mean \pm S.D. $(n=3) .{ }^{*} P<0.05$ vs. vehicle control. ${ }^{\#} P<0.05$ vs. si-USP14. h Western blot assay was used to detect the expression of PARP, Bcl-2, USP14, and HA-tag. GAPDH was used as an internal control. i MDAMB-453 cells were exposed to USP14 siRNA or control siRNA with or without AR or control vector for $72 \mathrm{~h}$. $\mathbf{j}$ MDA-MB-453 cells were exposed to IU1 with or without AR or control vector for $72 \mathrm{~h}$. MTS assay was used to detect cell viability. Error bars correspond to $95 \% \mathrm{CI}$ of three independent replicates. $* P<0.05$ vs. each vehicle control. ${ }^{\#} P$ $<0.05$ vs. si-USP14 or IU1 treatment

cancers. Blocking these receptors can lead to significant suppression of the development of many breast cancers. However, triple-negative breast cancer (TNBC), which is $\mathrm{ER}^{-}, \mathrm{PR}^{-}$, and $\mathrm{HER}^{-}$and comprised $15-20 \%$ of breast cancers, has the worst prognosis and vital organ metastases due to the lack of effective therapeutic targets [2, 33, 34]. Recent studies have identified AR as a novel therapeutic target in breast cancer, unveiling a great treatment opportunity for TNBC patients. Our current study showed that USP14, one of the $19 \mathrm{~S}$ proteasome-associated DUBs, stabilizes AR protein level by deubiquitinating the K48ubiquitin chain on AR. Loss of USP14 expression/function dramatically decreased AR level, blocked $G_{0} / G_{1}$ to $S$ phase transition, and triggered cell apoptosis in $\mathrm{AR}^{+}$breast cancer cells, suggesting that targeting USP14/AR axis could be a potential strategy for TNBC therapy.

Proteasomes recruit UCHL5 and USP14 to regulate the degradation of proteins. Inhibition of both UCHL5 and USP14 dramatically accumulates total K48-polyubiquitinated proteins $[30,31,35]$, suggesting that UCHL5 and USP14 are required for the proteasomemediated degradation of most proteins. However, the function of UCHL5 and USP14 for a specific protein remains largely unknown. Previously we identified USP14 as an AR DUB on $19 \mathrm{~S}$ proteasome by cleaving its Ubchain in LNCaP cells. The current study confirmed that USP14 removes the K48-ubiquitin chain on AR and subsequently inhibits proteasome-mediated degradation of AR, consistent with the finding that USP14 inhibited proteasome and mediated deubiquitination by reducing the anchoring duration of ubiquitin substrates on the proteasome [27, 28]. We wondered if UCHL5 exerts the same or opposite effects for AR. We found that dual inhibitors of USP14 and UCHL5 such as b-AP15 [31] and auranofin [30] reduced AR protein level in both prostate and breast cancer cells. We further explored the effect of UCHL5 knockdown on AR protein level and protein interaction between UCHL5 and AR. We found that UCHL5 silencing did not affect the expression of AR, and UCHL5 did not interact with AR via protein-protein binding under tested experimental condition, suggesting that USP14, but not UCHL5, is required to remove the ubiquitin chain on $\mathrm{AR}$ and stabilize $\mathrm{AR}$ protein on the $19 \mathrm{~S}$ particle.

DUBs have emerged as a class of novel therapeutic targets or biomarkers for anticancer strategies. Indeed, several DUBs have been reported to regulate AR expression or transcriptional activity. As an example, USP26 physically interacts with $\mathrm{AR}$ and influences $\mathrm{AR}$ ubiquitination and transcriptional activation [17]. Also, USP12 co-localizes with AR in the cytoplasm and promotes AR transcriptional activity by confronting the ubiquitin-dependent degradation of AR [15]. USP7 seems to be required to bind AR to chromatin, mediating its activity [16]. Moreover, USP10 not only binds AR, resulting in increased transcriptional activity, but also deubiquitinates the histone variant H2A.Z, both of which are required for $\mathrm{AR}^{-}$mediated gene activation [18, 19]. Therefore, our current study investigated the function of USP14 for AR translocation. We found that USP14 could not translocate AR into the nucleus under DHT stimulation. Nevertheless, loss of USP14 reduced the volume of nuclear AR under DHT stimulation, which may result from the reduction of cellular $A R$ after USP14 silencing.

We further investigated the molecular mechanisms by which USP14 contributes to breast cancer development and progression. USP14 inhibition or silencing significantly inhibits the growth of MDA-MB-453 and MDA-MB-231 breast cancer cells, and modestly suppresses the growth of breast cancer cells with either low AR expression (MDAMB-468 and HCC1937) or high AR/ER expression (MCF7). In addition, USP14 inhibition or silencing-induced $\mathrm{G}_{0} / \mathrm{G}_{1}$ arrest in MDA-MB 231, MDA-MB 453, and MCF7 breast cancer cells, associated with significantly reduced levels of CDK4, CDK2, and cyclin D1 proteins. This finding was consistent with findings from our previous study on prostate cancer, suggesting that USP14 plays an 
important role in cell cycle regulation. Moreover, inhibition or silencing of USP14 dramatically induced higher levels of cell death and PARP cleavage in MDA-MB 231 and MDAMB 453 than in MCF7 breast cancer cells. Surprisingly, treatment of USP14 inhibition or knockdown had no or little effect on breast cancer T47D cells that express high level of ER and very low level of AR. USP14 silencing-induced cell cycle arrest and apoptosis could be rescued by overexpressing AR, indicating that cell death and growth arrest are direct results of inhibition of AR deubiquitination. These findings also suggest that USP14 is definitely required for the growth and survival of $\mathrm{AR}^{+} / \mathrm{ER}^{-}$breast cancers.

In conclusion, this work has provided novel insights into the interaction between proteasome-associated DUB USP14 and $\mathrm{AR}$, and the functional role of deubiquitination of $\mathrm{AR}$ by USP14 in AR-positive human breast cancer cells including TNBC. Furthermore, the current research has provided a mechanistic base for targeting USP14 as a therapeutic strategy for treating patients with $\mathrm{AR}^{+} / \mathrm{ER}^{-}$ breast cancer.

\section{Materials and methods}

\section{Materials}

IU1 (catalog no. sc-361215), USP14 (sc-76817), AR (sc29204), and UCHL5 (c-76797) siRNAs were purchased from Santa Cruz Biotechnology (Santa Cruz, CA, USA). bAP15 (S4920), MG132 (S2619), and bortezomib (S1013) were purchased from Sellectchem (Houston, TX, USA). Aur (catalog no. BML-EI206-0100) was obtained from Enzo Life Sciences International, Inc. (Plymouth Meeting, PA). MTS (catalog no. G111) was from Promega Corporation (Madison, WI, USA). AnnexinV-FITC/PI apoptosis detection kits (KGA107) were purchased from Keygen Company (Nanjing, China). Co-IP assay kit (14311D) was obtained from Life Technologies (Carlsbad, CA). Antibodies were from the following corporations: anti-GAPDH (MB001), anti-CDK2 (BS1050), anti-GFP (BSAP0675M), anti-Bcl-2 (BS70205) (Bioworld Technology, Inc., Louis Park, MN, USA); anti-Ubiquitin (catalog no. 3936), antiPARP (9532), anti-CDK4 (12790), anti-USP14 (11931), anti-Lamin B (13435), anti-HSP90 (4877), HA-tag (3724), anti-K48-ub (12805), anti-cyclin D1 (2922) (Cell Signaling Technology, Beverly, MA, USA); anti-AR (ab108341), anti-UCHL5 (ab124931) (Abcam, USA).

\section{Cell lines and cell cultures}

The following cell lines are from American Type Culture Collection (Manassas, VA, USA): MDA-MB-453, MDA-
MB-231, MCF7, MDA-MB 468, HCC1937, T47D, and LNCaP. HCC1937 and LNCaP were grown in RPMI 1640, $10 \%$ fetal bovine serum (FBS) and penicillin/streptomycin. MDA-MB 453, MDA-MB 231, MCF7, MDA-MB 468, and T47D cells were grown in HyClone DMEM, 10\% FBS and penicillin/streptomycin.

\section{Cell viability assay}

Cell viability was detected using MTS assay (CellTiter 96Aqueous One Solution reagent) as we previously reported [36]. Briefly, cultured MDA-MB-453, MDA-MB-231, MCF7, MDA-MB-468, HCC1937, or T47D cells were digested and suspended at $2 \times 10^{4}$ cells $/ \mathrm{ml}$ medium, and then randomly seeded with $100 \mu$ cell suspensions in 96well plate. The cells were incubated for $24 \mathrm{~h}$ and then exposed to IU1 or USP14 shRNA for one to 5 days. MTS reagent was added to each well. After $3 \mathrm{~h}$ reaction at $37^{\circ}$, the absorbance of density of each well was read at a wavelength of $490 \mathrm{~nm}$ with a microplate reader (Sunrise, Tecan).

\section{Flow cytometry analysis of cell cycle and apoptosis}

For cell cycle assay, the indicated breast cancer cells were exposed to IU1 or USP14/control shRNA for $48 \mathrm{~h}$. Cells were collected and then washed three times with $4^{\circ} \mathrm{C} \mathrm{PBS}$, and then resuspended with $500 \mu \mathrm{l}$ PBS plus $2 \mathrm{ml} 70 \%$ ethanol at $4^{\circ} \mathrm{C}$ for $12 \mathrm{~h}$. Cells were then centrifuged and washed with $4^{\circ} \mathrm{C}$ PBS again, followed by $50 \mu \mathrm{g} / \mathrm{ml}$ PI, 100 $\mu \mathrm{g} / \mathrm{ml}$ RNaseA, $0.2 \%$ Triton-X-100 complex incubation for $30 \mathrm{~min}$ at $4^{\circ} \mathrm{C}$. The flow cytometry was used to analyze the stained cells. Cell apoptosis analysis was performed as we previously described [37]. Briefly, the indicated breast cancer cells were exposed to IU1 or USP14/control siRNA for $72 \mathrm{~h}$. Cells were collected and washed three times with $4^{\circ} \mathrm{C}$ PBS. Then cells were resuspended with $500 \mu \mathrm{l}$ annexinV-FITC binding buffer, $5 \mu \mathrm{l}$ annexinV-FITC, and 5 $\mu \mathrm{l}$ PI mixture in each group. After incubation for $30 \mathrm{~min}$, flow cytometry was used to analyze the stained cells.

\section{Clonogenic assay}

Clonogenic assay was performed as previously described [38]. The indicated breast cancer cells were exposed to IU1 or USP14/control shRNA for $48 \mathrm{~h}$. Then the cells in each group were digested, resuspended and randomly seeded in $60 \mathrm{~mm}$ dishes supplemented with 10\% FBS DMEM medium, cultured in an atmosphere of $5 \% \mathrm{CO}_{2}$ for 2 weeks. Cells were fixed with $4 \%$ paraformaldehyde for $15 \mathrm{~min}$, then washed with PBS twice, followed by crystal violet solution incubation for $5 \mathrm{~min}$. Colonies $>60 \mu \mathrm{m}$ were counted from three independent repeated experiments. 


\section{siRNA transfection}

SiRNA transfection was performed as previously described [39]. Briefly, the indicated breast cancer cells were randomly seeded in $60 \mathrm{~mm}$ dishes for $24 \mathrm{~h}$. RPMI opti-MEM, lipofectamine RNAiMax (Invitrogen) reagent and siRNAs (Santa Cruz, CA) targeting human USP14/UCHL5/AR or siRNAs(Santa Cruz, CA) with non-specific sequences mixtures were prepared respectively, then the mixtures was added in each group and cultured for $72 \mathrm{~h}$. Fresh medium was replaced appropriately after transfection for $6 \mathrm{~h}$.

\section{Lentivirus USP14 shRNA transfection}

Lentivirus (pLent-4in1shRNA-GFP) containing human USP14 (NM-005151) shRNA or control shRNA was purchased from VigeneBio (Shandong, China). Exponentially growing cells were randomly seeded in $60 \mathrm{~mm}$ dishes. When the cells were cultured overnight and reached 50\% confluence, medium containing lentiviruses and polybrene $(5 \mu \mathrm{g} / \mathrm{ml}$; Santa Cruz, CA, USA) was added at a multiplicity of infection of 10 and mixed with the cells. After overnight incubation, supernatant in each well was replaced with DMEM containing 10\% FBS and cultured for $48 \mathrm{~h}$. For selection of stably-transfected cells, we proceeded with puromycin selection performed by aspirating the medium and replacing it with fresh medium containing puromycin (Santa Cruz, CA, USA) at the concentration of $2 \mu \mathrm{g} / \mathrm{ml}$ and then aspirating and replacing with freshly prepared selective medium and culturing the surviving cells approximately every 2 days.

\section{RNA extraction and PCR assay}

RNAs were extracted from MDA-MB 453 cells treated with IU1 or USP14/control shRNA for $24 \mathrm{~h}$ with RNAiso plus (TaKaRa Biotechnology, Dalian, China) and performed according to the manufacturer's instructions. The Purity and concentration of RNAs in each sample were read with 260:280 nm. The First-strand cDNA was synthesized with $1 \mu \mathrm{g}$ total RNA and the use of PrimeScript RT Master Mix kit (TaKaRa, Dalian, China). Real-time quantitative PCR was used to measure the mRNA expression levels of AR. GAPDH, a housekeeping gene, was here used as an internal control. SYBR Premix Ex Taq ${ }^{\mathrm{TM}}$ kit (TaKaRa, Dalian, China) was used according to the manufacturer's instructions. The primers in this study for PCR were as follow, AR: F: 5'-GGTGAGCAGAGTGCCCTATC-3'; R: 5'GAAGAC CTTGCAGCTTCCAC-3'; GAPDH: F:5'TCCCATCACCATCTTCCA-3'; R: 5'-CATCACGCCACAGTTTCC-3'.

\section{Protein expression and interaction analysis}

Protein interaction (Co-IP) analysis was performed as described in a previous study [11]. In brief, antibodies and dynabeads (Invitrogen) mixtures were prepared overnight. Then the cell lysates extracted from MDA-MB 453 were added in the antibodies-beads mixtures. After incubation and rotation at $4^{\circ} \mathrm{C}$ for $1 \mathrm{~h}$, the antibodies-prays mixtures were washed with PBS-T for three times. Then the mixtures were suspended with appropriate SDS loading buffer and separated from dynabeads. Western blot was used to analyze protein expression. This assay was performed as described previously [40].

\section{Immunofluorescence microscopy}

Cells stably expressing USP14 or scramble shRNA were exposed to DHT $10 \mathrm{nM}$ for $24 \mathrm{~h}$. Then $4 \%$ paraformaldehyde was used to fixed cells for $15 \mathrm{~min}$. $0.5 \%$ Triton-X was used to permeabilize cells for $5 \mathrm{~min}$. 5\% BSA (bovine serum albumin, Sigma) was used to block for $30 \mathrm{~min}$. And then the cells were incubated with AR primary antibodies (Abcam) overnight at $4^{\circ} \mathrm{C}$. Next the cells were incubated with anti-rabbit IgG-cy3 secondary antibodies (Bioworld) for $1 \mathrm{~h}$. DAPI (4',6-diamidino-2-phenylindole, Abcam) was used to indicate the nucleus. Images were acquired using an Olympus fluorescence microscope with $\times 400$ magnification.

\section{Plasmids and transfection of cells}

The plasmid HA-AR encoding a fusion protein of AR and HA-tag or control vector was purchased from Genechem (Shanghai, China). Exponentially growing MDA-MB-453 cells stably expressing USP14 or scramble shRNA were seeded in six-well plates for $24 \mathrm{~h}$. The cells were transfected with plasmid HA-AR or control vector mixed with lipofectamine 2000 reagent (Life Technologies). Then the cells were incubated for $48 \mathrm{~h}$ for further analysis.

\section{Statistical methods}

Data are presented as mean \pm SD from three independent experiments where applicable. To determine statistical probabilities, Unpaired Student's $t$ test or one way ANOVA is used where appropriate. Statistical analysis was performed by GraphPad Prism5.0 software (GraphPad Software) and SPSS 16.0. A $P$ value of $>0.05$ was considered statistically significant.

Acknowledgments We thank Christine Heiner (Department of Surgery, University of Pittsburgh) for her critical reading of the manuscript. The study was supported by the National Natural Science 
Foundation of China (81472390, 81472762, and 31671435), the National Funds for Developing Local Colleges and Universities (B16056001), the Science and Technology Program of Guangzhou (201604020001), Pearl River S\&T Nova Program of Guangzhou (201506010071), Innovative Academic Team of Guangzhou Education System (1201610014), General Project (1201610098) from Guangzhou Education Commission, the Science and Technology Planning Project of Guangdong Province, China (2014A020212691 and 2016A030308), a Research Award Fund for Outstanding Young Teachers in Guangdong Provincial Higher Education Institutions (YQ2015136), the Project of Department of Education of Guangdong Province(2016KTSCX118, 2016KTSCX119), a Research Scholar Grant from the American Cancer Society (RSG-16-014-01-CDD), the National Institutes of Health, USA (R01CA160417 and R01GM115366), the Natural Science Foundation of Guangdong Province (2016A030308011), and Guangdong Province Universities and Colleges Pearl River Scholar Funded Scheme (2017). We thank the Guangdong Provincial Key Laboratory of Malignant Tumor Epigenetics and Gene Regulation, Sun Yat-Sen Memorial Hospital, Sun Yat-Sen University for flow cytometry analysis.

\section{Compliance with ethical standards}

Conflict of interest The authors declare that they have no competing interests.

Open Access This article is licensed under a Creative Commons Attribution-NonCommercial-ShareAlike 4.0 International License, which permits any non-commercial use, sharing, adaptation, distribution and reproduction in any medium or format, as long as you give appropriate credit to the original author(s) and the source, provide a link to the Creative Commons license, and indicate if changes were made. If you remix, transform, or build upon this article or a part thereof, you must distribute your contributions under the same license as the original. The images or other third party material in this article are included in the article's Creative Commons license, unless indicated otherwise in a credit line to the material. If material is not included in the article's Creative Commons license and your intended use is not permitted by statutory regulation or exceeds the permitted use, you will need to obtain permission directly from the copyright holder. To view a copy of this license, visit http://creativecommons. org/licenses/by-nc-sa/4.0/.

\section{References}

1. Di Cosimo S, Baselga J. Management of breast cancer with targeted agents: importance of heterogeneity. Nat Rev Clin Oncol. 2010;7:139-47.

2. Qin J, Zhou Z, Chen W, Wang C, Zhang H, Ge G, et al. BAP1 promotes breast cancer cell proliferation and metastasis by deubiquitinating KLF5. Nat Commun. 2015;6:8471.

3. Ni M, Chen Y, Lim E, Wimberly H, Bailey ST, Imai Y, et al. Targeting androgen receptor in estrogen receptor-negative breast cancer. Cancer Cell. 2011;20:119-31.

4. Collins LC, Cole KS, Marotti JD, Hu R, Schnitt SJ, Tamimi RM. Androgen receptor expression in breast cancer in relation to molecular phenotype: results from the Nurses' Health Study. Mod Pathol. 2011;24:924-31.

5. Guedj M, Marisa L, de Reynies A, Orsetti B, Schiappa R, Bibeau $\mathrm{F}$, et al. A refined molecular taxonomy of breast cancer. Oncogene. 2012;31:1196-206.
6. Peters AA, Buchanan G, Ricciardelli C, Bianco-Miotto T, Centenera MM, Harris JM, et al. Androgen receptor inhibits estrogen receptor-alpha activity and is prognostic in breast cancer. Cancer Res. 2009;69:6131-40.

7. Cochrane DR, Bernales S, Jacobsen BM, Cittelly DM, Howe EN, D'Amato NC, et al. Role of the androgen receptor in breast cancer and preclinical analysis of enzalutamide. Breast Cancer Res. 2014;16:R7.

8. Doane AS, Danso M, Lal P, Donaton M, Zhang L, Hudis C, et al. An estrogen receptor-negative breast cancer subset characterized by a hormonally regulated transcriptional program and response to androgen. Oncogene. 2006;25:3994-4008.

9. Gucalp A, Tolaney S, Isakoff SJ, Ingle JN, Liu MC, Carey LA, et al. Phase II trial of bicalutamide in patients with androgen receptor-positive, estrogen receptor-negative metastatic Breast Cancer. Clin Cancer Res. 2013;19:5505-12.

10. Gonzalez-Angulo AM, Stemke-Hale K, Palla SL, Carey M, Agarwal R, Meric-Berstam F, et al. Androgen receptor levels and association with PIK3CA mutations and prognosis in breast cancer. Clin Cancer Res. 2009;15:2472-78.

11. Liao Y, Liu N, Hua X, Cai J, Xia X, Wang X, et al. Proteasomeassociated deubiquitinase ubiquitin-specific protease 14 regulates prostate cancer proliferation by deubiquitinating and stabilizing androgen receptor. Cell Death Dis. 2017;8:e2585.

12. Lin HK, Wang L, Hu YC, Altuwaijri S, Chang C. Phosphorylation-dependent ubiquitylation and degradation of androgen receptor by Akt require $\mathrm{Mdm} 2 \mathrm{E} 3$ ligase. EMBO J. 2002;21:4037-48.

13. Qi J, Tripathi M, Mishra R, Sahgal N, Fazli L, Ettinger S, et al. The E3 ubiquitin ligase Siah2 contributes to castration-resistant prostate cancer by regulation of androgen receptor transcriptional activity. Cancer Cell. 2013;23:332-46.

14. Sarkar S, Brautigan DL, Parsons SJ, Larner JM. Androgen receptor degradation by the E3 ligase CHIP modulates mitotic arrest in prostate cancer cells. Oncogene. 2014;33:26-33.

15. Burska UL, Harle VJ, Coffey K, Darby S, Ramsey H, O'Neill D, et al. Deubiquitinating enzyme Usp12 is a novel co-activator of the androgen receptor. J Biol Chem. 2013;288:32641-50.

16. Chen ST, Okada M, Nakato R, Izumi K, Bando M, Shirahige K. The deubiquitinating enzyme USP7 regulates androgen receptor activity by modulating its binding to chromatin. J Biol Chem. 2015;290:21713-23.

17. Dirac AM, Bernards R. The deubiquitinating enzyme USP26 is a regulator of androgen receptor signaling. Mol Cancer Res. 2010;8:844-54.

18. Draker R, Sarcinella E, Cheung P. USP10 deubiquitylates the histone variant H2A.Z and both are required for androgen receptor-mediated gene activation. Nucleic Acids Res. 2011;39:3529-42.

19. Faus H, Meyer HA, Huber M, Bahr I, Haendler B. The ubiquitinspecific protease USP10 modulates androgen receptor function. Mol Cell Endocrinol. 2005;245:138-46.

20. Mialki RK, Zhao J, Wei J, Mallampalli DF, Zhao Y. Overexpression of USP14 protease reduces I-kappaB protein levels and increases cytokine release in lung epithelial cells. J Biol Chem. 2013;288:15437-41.

21. Sahtoe DD, van Dijk WJ, El Oualid F, Ekkebus R, Ovaa H, Sixma TK, Mechanism of UCH-L5 activation and inhibition by DEUBAD domains in RPN13 and INO80G. Mol Cell. 2015;57:887-900.

22. Tian Z, D'Arcy P, Wang X, Ray A, Tai YT, Hu Y, et al. A novel small molecule inhibitor of deubiquitylating enzyme USP14 and UCHL5 induces apoptosis in multiple myeloma and overcomes bortezomib resistance. Blood. 2014;123:706-16. 
23. Shinji S, Naito Z, Ishiwata S, Ishiwata T, Tanaka N, Furukawa K, et al. Ubiquitin-specific protease 14 expression in colorectal cancer is associated with liver and lymph node metastases. Oncol Rep. 2006;15:539-43.

24. Wang Y, Wang J, Zhong J, Deng Y, Xi Q, He S, et al. Ubiquitinspecific protease 14 (USP14) regulates cellular proliferation and apoptosis in epithelial ovarian cancer. Med Oncol. 2015;32:379.

25. Borodovsky A, Kessler BM, Casagrande R, Overkleeft HS, Wilkinson KD, Ploegh HL. A novel active site-directed probe specific for deubiquitylating enzymes reveals proteasome association of USP14. EMBO J. 2001;20:5187-96.

26. Hu M, Li P, Song L, Jeffrey PD, Chenova TA, Wilkinson KD, et al. Structure and mechanisms of the proteasome-associated deubiquitinating enzyme USP14. EMBO J. 2005;24:3747-56.

27. Lee BH, Lee MJ, Park S, Oh DC, Elsasser S, Chen PC, et al. Enhancement of proteasome activity by a small-molecule inhibitor of USP14. Nature. 2010;467:179-84.

28. Lee BH, Lu Y, Prado MA, Shi Y, Tian G, Sun S, et al. USP14 deubiquitinates proteasome-bound substrates that are ubiquitinated at multiple sites. Nature. 2016;532:398-401.

29. McClurg UL, Robson CN. Deubiquitinating enzymes as oncotargets. Oncotarget. 2015;6:9657-68.

30. Liu N, Li X, Huang H, Zhao C, Liao S, Yang C, et al. Clinically used antirheumatic agent auranofin is a proteasomal deubiquitinase inhibitor and inhibits tumor growth. Oncotarget. 2014;5:5453-71.

31. D’Arcy P, Brnjic S, Olofsson MH, Fryknas M, Lindsten K, De Cesare M, et al. Inhibition of proteasome deubiquitinating activity as a new cancer therapy. Nat Med. 2011;17:1636-40.
32. Yuan J, Luo K, Zhang L, Cheville JC, Lou Z. USP10 regulates p53 localization and stability by deubiquitinating p53. Cell. 2010;140:384-96.

33. Elias AD. Triple-negative breast cancer: a short review. Am J Clin Oncol. 2010;33:637-45.

34. Hurvitz SA, Finn RS. What's positive about 'triple-negative' breast cancer? Future Oncol. 2009;5:1015-25.

35. Huang H, Liao Y, Liu N, Hua X, Cai J, Yang C, et al. Two clinical drugs deubiquitinase inhibitor auranofin and aldehyde dehydrogenase inhibitor disulfiram trigger synergistic anti-tumor effects in vitro and in vivo. Oncotarget. 2016;7:2796-808.

36. Huang H, Chen D, Li S, Li X, Liu N, Lu X, et al. Gambogic acid enhances proteasome inhibitor-induced anticancer activity. Cancer Lett. 2011;301:221-28.

37. Huang H, Zhang X, Li S, Liu N, Lian W, McDowell E, et al. Physiological levels of ATP negatively regulate proteasome function. Cell Res. 2010;20:1372-85.

38. Shi X, Chen X, Li X, Lan X, Zhao C, Liu S, et al. Gambogic acid induces apoptosis in imatinib-resistant chronic myeloid leukemia cells via inducing proteasome inhibition and caspasedependent Bcr-Abl downregulation. Clin Cancer Res. 2014;20:151-63.

39. Huang H, Hua X, Liu N, Li X, Liu S, Chen X, et al. Anacardic acid induces cell apoptosis associated with induction of ATF4dependent endoplasmic reticulum stress. Toxicol Lett. 2014;228:170-78.

40. Huang H, Liu N, Guo H, Liao S, Li X, Yang C, et al. L-carnitine is an endogenous HDAC inhibitor selectively inhibiting cancer cell growth in vivo and in vitro. PLoS ONE. 2012;7:e49062. 